\title{
Low Surface Brightness Optical Emission as a Probe of Fossil Cosmic Ray Reservoirs
}

\section{W. Thomas Vestrand*}

Los Alamos National Laboratory

P.O. Box 1663

Los Alamos, NM 87545 USA

E-mail: vestrandelanl.gov

\section{Lucas Parker}

Los Alamos National Laboratory

P.O. Box 1663

Los Alamos, NM 87545 USA

E-mail: lpp@lanl.gov

\begin{abstract}
New techniques for detecting low surface brightness optical features are emerging that are enabling previous impossible observations. We briefly discuss the basis for this new approach that employs arrays of telephoto lenses and describe an array that is nearing completion at Los Alamos National Laboratory. We then explore the case for fossil cosmic-ray electron reservoirs in places where the traditional signatures of current activity are not present and discuss how optical inverse Compton emission can be used to search for fossil reservoirs
\end{abstract}

36th International Cosmic Ray Conference -ICRC2019-

July 24th - August 1st, 2019

Madison, WI, U.S.A.

${ }^{*}$ Speaker. 


\section{Introduction}

In the last five years, advances in commercial off-the-shelf (COTS) telephoto lenses and astronomical image processing techniques have come together in an unconventional approach to telescope design that has disrupted the field of low-surface brightness optical astronomy and pushed the previous surface brightness limit from where it had stagnated for many decades to a new limit that is nearly 100x fainter. The impressive scientific returns from the first applications of this new telescope design approach [1], which employs arrays of telephoto lenses, suggests that low surface brightness observations will be a rich area of discovery for a broad range of predicted, but until now mostly unobservable, astrophysical phenomena. The ultimate limits of this new approach are unknown, but there is already speculation [2] that it could scale to a 500-lens array capable of directly imaging diffuse ionized gas in the intergalactic medium - the most important and least understood baryonic component of the Universe!

In this paper we present a description of this new telescope design approach as well as an array using those principles that is nearing completion at Los Alamos National Laboratory. We then briefly discuss the prospect of using low surface brightness optical imaging to explore questions of interest to the cosmic ray community. In particular, we examine the possibility of using optical inverse Compton emission as a probe of fossil cosmic ray electron reservoirs.

\section{Optical Low Surface Brightness Imaging}

Most astronomical telescope designs employ reflecting optics and are optimized by employing the largest aperture mirrors possible to provide the highest spatial resolution and sensitivity for point-like objects. But the price of that optimization is scattered light into the wings of the optical point spread function (PSF) (generated by complicated, folded, optical paths, by diffraction from secondary mirror support structure, etc.) that hide low surface brightness objects. For low surface brightness studies, you must design to suppress the power in the wings of the PSF and optimize the "speed" of the optics in order to maximize the number of detected photons per camera pixel. The figure of merit for that optimization scales as $\sim \varepsilon \mathrm{Af}^{-2}$ (where $\mathrm{A}$ is the camera pixel area, $\varepsilon$ is the detection quantum efficiency, and $\mathrm{f}$ is the telescope focal ratio (focal length/aperture diameter)) - so one designs for a low $f$ ratio ("optically fast") telescope instead of a large aperture.

Our LANL system is using an approach to low surface brightness imaging, pioneered by the Dragonfly Team [1], which employs arrays of co-aligned telephoto lenses. Driven by the demands of the highly competitive digital photography industry, commercial telephoto lenses are the highest optical quality and physically robust fast "telescopes" in existence. And Canon has invented a technology called Sub-Wavelength Structure (SWS) coatings that gives their new generation of telephoto lenses PSF wings that are significantly smaller than conventional optics. This technology is a game changer for the construction of low surface brightness imagers.

The LANL low surface array employs 16 Canon 400mm 2.8 II lenses that are co-aligned and deployed on a single fully robotic telescope mount. On the basis of functionality, the full array can be divided into two telescope sub-arrays: (1) An eight telescope narrow field, high spatial resolution array; and (2) an eight camera wide-field, high sensitivity (but lower spatial resolution) array. The narrow-field sub-array uses prosumer-class STF-8300M CCD cameras from Santa Barbara 
Imaging Group that employ front-illuminated Kodak CCD chips with $5.4 \mu \mathrm{m}$ pixels. The field-ofview for this sub-array is $1.9^{\circ} \times 2.6^{\circ}$ and each pixel corresponds to 2.8 arcsec on the sky — roughly equal to the typical seeing at the site. The wide-field sub-array employs higher quality, large format, scientific-grade CCD cameras from Andor Technology Ltd. which give it a $\left(4^{\circ} \times 4^{\circ}\right)$ field-of-view that is approximately four times larger than the high-resolution sub-array. The cameras also employ $2 \mathrm{Kx} 2 \mathrm{~K}$ pixel E2V back-illuminated CCD chips that have nearly twice the quantum detection efficiency (92\% versus $48 \%$ at $650 \mathrm{~nm}$ ) and a CCD pixel area that is $\sim 4 \times$ larger. Altogether the wide-field array has a low surface brightness imaging figure-of-merit that is a factor of $\sim 6$ greater than the high resolution array. However, the larger pixel areas of the wide-field cameras also increases the potential for the detection of false positives generated by source confusion (blending) in crowded fields. To mitigate that risk our operational discovery procedure for low surface brightness features involves identification of candidate features with the wide-field sub-array and follow-up verification with the high-resolution sub-array.

Software also plays an essential role in enabling sensitive low-surface brightness imaging. The cameras in the array are all slaved together to collect synchronized exposures-typically of 4 minutes duration - and each of the eight images from a given sub-array is translated, rotated and scaled to allow them to be co-added into a single image. All cameras in the array have dark current and read noise that is smaller than the shot noise from the sky background and all employ identical autofocus software. In this case, the co-added image from $\mathrm{N}$ identical lenses with aperture $\mathrm{D}$ and focal ratio $\mathrm{f}$, is essentially equivalent to the image from a single conventional telescope with an effective aperture $D_{\text {eff }}=N^{1 / 2} D$ and effective focal ratio $f_{\text {eff }}=f \times N^{-1 / 2}$. Then a series of those co-added images will be similarly "stacked" into an image with a much longer $(\sim 50-100$ hour) effective exposure, that is more sensitive for the detection of low-surface brightness features. Altogether, this approach allows lens arrays to act as ultra-fast astronomical telescopes that are impossible to build using traditional design approaches.

\section{Formation of Fossil Reservoirs}

We expect that the generation of comic rays started very early in the history of the Universe with the deaths of the first generation stars. And the detection of high redshift gamma-ray bursts indicates that high energy particles were being generated only 630 million years after the Big Bang [3]. The supermassive black holes that power Active Galactic Nuclei (AGN) also erupt into powerful outbursts of particle acceleration and then lapse into periods of quiescence. The long history and episodic nature of cosmic ray particle generation leads to the question: Is there fossil evidence of previous particle acceleration in places where the signatures of current activity are not present? To explore that question we need to examine: (1) How long do cosmic rays of various energies live?; (2) What observable signatures do the long-lived particles generate?; and (3) How could they accumulate in fossil reservoirs where they would be easier to detect?

\subsection{Particle Lifetimes}

In our galaxy, the so-called Ionization losses dominate other energetic electron loss mechanisms below $\sim 20 \mathrm{MeV}$. In the intracluster medium of a galaxy cluster or the intercluster medium between galaxy clusters, where the gas is a dilute and fully ionized plasma, relativistic electrons no 


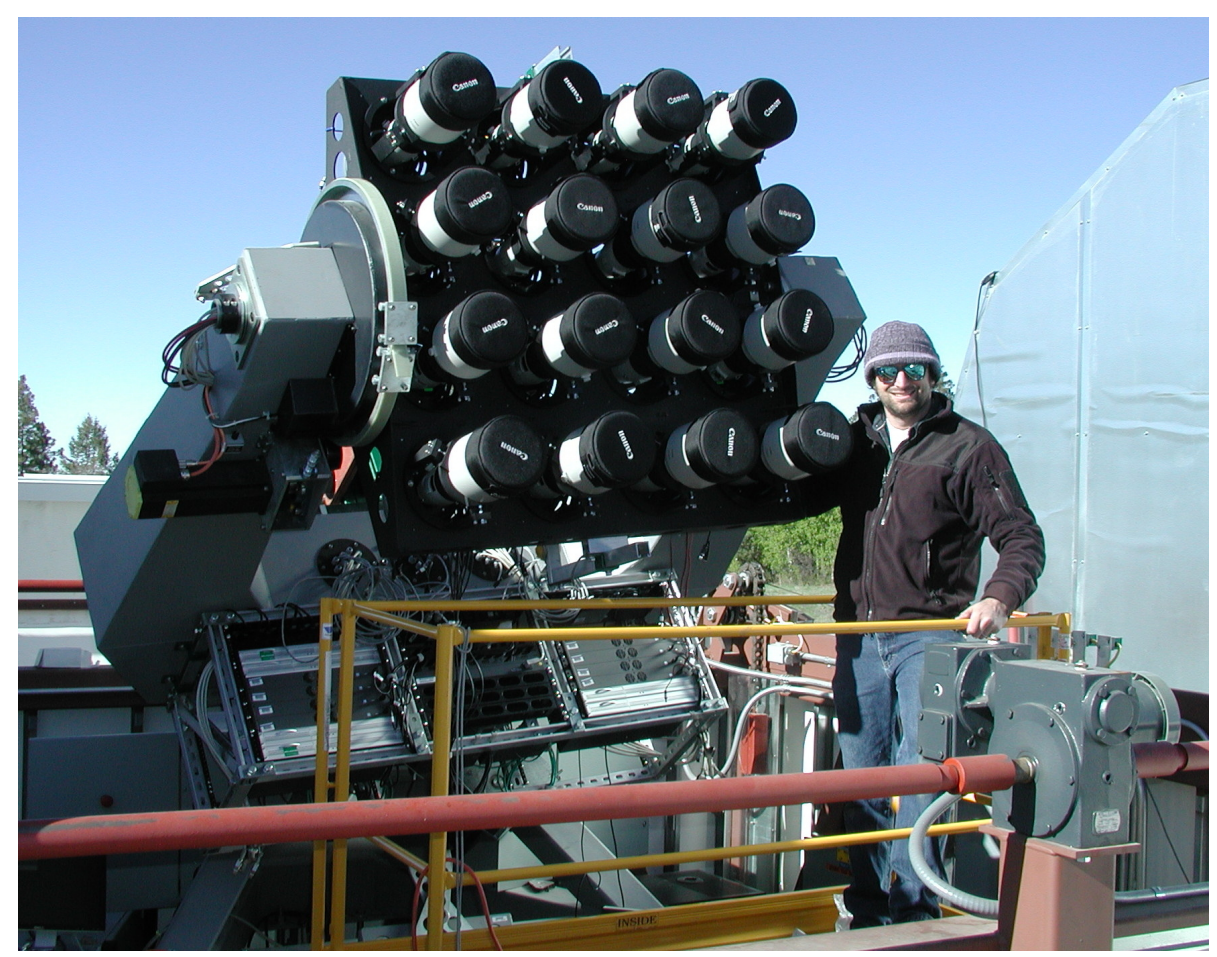

Figure 1: The LANL low surface brightness array employs sixteen, co-alined, Canon 400mm F2.8 lenses to collect images that are stacked to form a deeper image that is optimized for low surface brightness imaging. The array is deployed at a high altitude $(8,750 \mathrm{ft}$.), light pollution free site in the Jemez Mountains of Northern New Mexico.

longer lose energy by exciting and ionizing atoms. Instead, the electrons lose energy by the excitation of electron plasma oscillations. The physics of this process has been examined in great detail by Gould [4]. His equation for the loss rate can be rewritten in practical units for the intracluster medium and turned into a characteristic energetic electron lifetime of:

$T_{\text {Ion }}=\gamma_{e}\left(d \gamma_{e} / d t\right)_{\text {Ion }}^{-1}=3.5 \times 10^{8}\left(\frac{\mathrm{n}_{\mathrm{e}}}{3 \times 10^{-3} \mathrm{~cm}^{-3}}\right)^{-1}\left(40+\ln \left(\gamma_{e}^{1 / 2}\left(\frac{\mathrm{n}_{\mathrm{e}}}{3 \times 10^{-3} \mathrm{~cm}^{-3}}\right)^{1 / 2}\right)\right)^{-1} \gamma_{e} \mathrm{yrs}$

where $\gamma_{e} \mathrm{mc}^{2}$ is the energy of the cosmic ray electron and $n_{e}$ is the number density of the plasma.

The bremsstrahlung losses are produced by the inelastic collisions of the cosmic ray electrons with ions and electrons in the plasma. In a hot intracluster medium the "no screening" approximation is quite accurate for calculating the bremsstrahlung loss. So the classic formula [5] can be translated into a characteristic lifetime of:

$$
T_{\text {Brem }}=\gamma_{e}\left(d \gamma_{e} / d t\right)_{\text {Brem }}^{-1}=7.8 \times 10^{10}\left(\frac{\mathrm{n}_{\mathrm{e}}}{3 \times 10^{-3} \mathrm{~cm}^{-3}}\right)^{-1}\left(\ln \gamma_{e}+0.36\right)^{-1} \text { years }
$$

At high energies the losses from synchrotron radiation becomes important. When a cosmic ray electron with an energy $\gamma_{e} \mathrm{mc}^{2}$ gyrates in a magnetic field with strength $\mathrm{B}$, it radiates energy at a rate [6] that leads to a characteristic loss timescale of:

$$
T_{S y n}=\gamma_{e}\left(d \gamma_{e} d t\right)_{S y n}^{-1}=2.5 \times 10^{13}\left(\frac{\mathrm{B}}{1 \mu \mathrm{G}}\right)^{-2} \gamma_{e}^{-1} \text { years }
$$


But in locations where the magnetic field strength B is less than $3.2 \times 10^{-6}$ Gauss, the most important loss mechanism for very high energy electrons is the inverse Compton process [7]. Here the relativistic electron collides with a low energy photon, producing a high-energy recoil photon and a corresponding decrease in the electron energy. If the energy density of the ambient photons is $\rho_{\text {rad }}$, the characteristic loss can be written as:

$$
T_{I C}=\gamma_{e}\left(d \gamma_{e} / d t\right)_{I C}^{-1}=2.4 \times 10^{12}\left(\frac{\rho_{\text {rad }}}{0.25 \mathrm{eV} \mathrm{cm}^{-3}}\right)^{-1} \gamma_{e}^{-1} \text { years }
$$

Here the baseline radiation field density is the Cosmic Microwave Background (CMB) value of $0.25 \mathrm{eV} \mathrm{cm}^{-3}$.
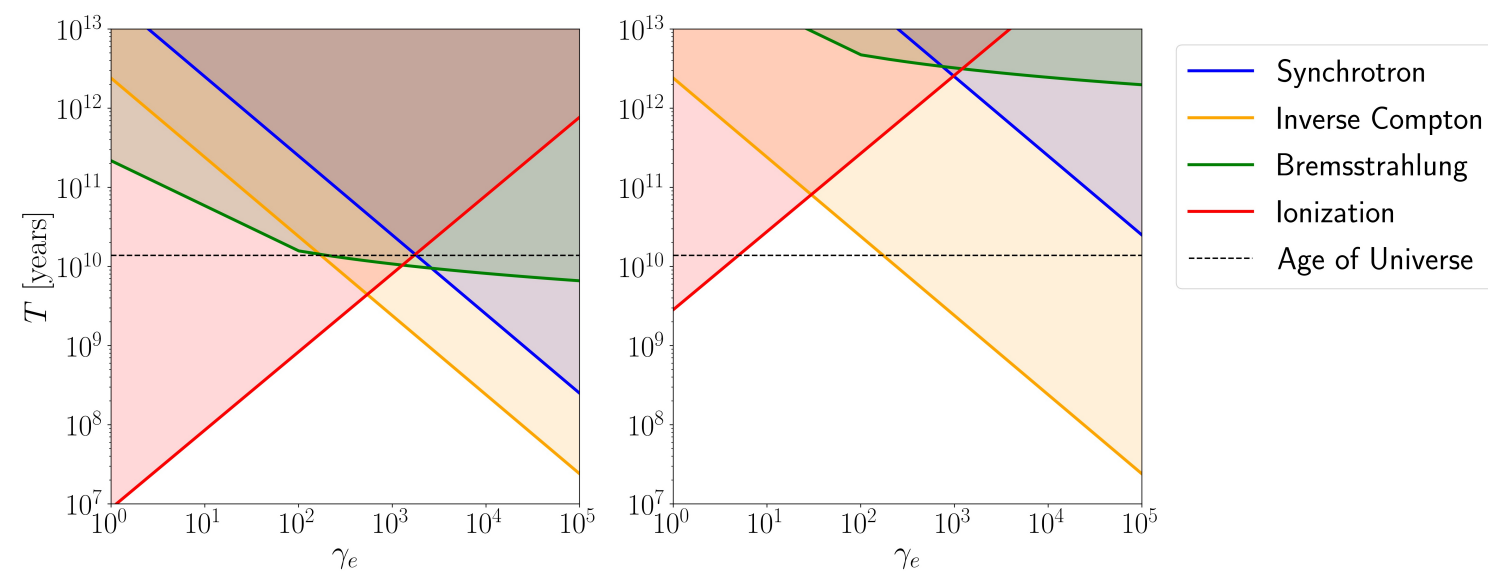

Figure 2: The characteristic loss timescales for cosmic ray electrons with energy $\gamma_{e} \mathrm{mc}^{2}$ are very dependent on the local environmental conditions. The left-hand panel shows the loss timescales for the conditions $\left(n_{e}=3 \times 10^{-3} \mathrm{~cm}^{-3}, \mathrm{~B}=1 \mu \mathrm{G}\right)$ typically found in the intracluster medium of a rich cluster of galaxies. It clear that none of the energetic electrons generated in the early Universe could survive until today in that environment. The right-hand panel shows the loss timescales for conditions $\left(n_{e}=10^{-5} \mathrm{~cm}^{-3}, \mathrm{~B}=0.1 \mu \mathrm{G}\right)$ between clusters in a Supercluster. In that environment, electrons with $\gamma_{e} \sim 50$ survive longer than the current age of the Universe.

It is instructive to jointly plot these four characteristic loss timescales for the physical conditions of potential fossil reservoir locations. The constraints on cosmic ray streaming that we discuss in the next section, suggest that galaxy clusters or superclusters are likely candidates for fossil reservoir residence. Typical conditions in the intracluster medium of a rich cluster of galaxies are $n_{e}=3 \times 10^{-3} \mathrm{~cm}^{-3}, B=1 \mu G$ and the CMB is the dominant radiation energy density. Recent work on the gas density between clusters[8] in superclusters suggests a much lower intercluster density of $n_{e} \simeq 10^{-5} \mathrm{~cm}^{-3}$. Plotting the loss timescales for those conditions (figure 2) shows that the longest lived intracluster particles are those with $\gamma_{e} \simeq 500$ and lifetimes of only a few Billion years. In the lower density supercluster intercluster medium, the peak lifetime shifts down to $\gamma_{e} \simeq 30$. But those cosmic ray electrons and even those at $\gamma_{e} \simeq 100$ live longer than the age of the Universe. This intercluster gas in superclusters is therefore the ideal environment for housing fossil cosmic ray electron reservoirs as are the Radio Ghost regions in clusters that have been proposed by Ensslin [9]. The principal loss mechanism and potentially observable signature of these fossil particles is the radiating of inverse Compton emission. 


\subsection{Optical Emission from Fossil Cosmic Ray Reservoirs}

The spectral energy distribution of the radiated inverse Compton emission is strongly peaked around an average upscattered photon frequency of $\langle v\rangle=4 / 3 \gamma_{e}^{2} v_{o}$ where $v_{o}$ is the typical frequency of the seed photons. The optical band spans frequencies from $4.3 \times 10^{14} \mathrm{~Hz}$ to $7.7 \times$ $10^{14} \mathrm{~Hz}$. For CMB seed photons, the mean photon frequency is $v_{o}=1.55 \times 10^{11} \mathrm{~Hz}$. So it is easy to see that the optical band is optimal for finding fossil cosmic ray electrons with $\gamma_{e} \sim 50$. The inverse Compton power radiated by a fossil energetic electron is given by [7]

$$
P_{I C}=4 / 3 \sigma_{T} c \gamma_{e}^{2} \rho_{\text {rad }}
$$

where $\sigma_{T}=6.65 \times 10^{-25} \mathrm{~cm}^{-2}$ is the Thomson cross section of an electron.

\subsection{Sources and Trapping}

The generation of fossil reservoirs depends on the unknown history of cosmic ray acceleration in the early Universe. However, a hint that fossil reservoirs might exist is provided by a class of radio sources called radio relics[10]. These elongated or irregular shaped radio sources are found in the outskirts of galaxy clusters and have no clear association with an active radio galaxy or AGN. The original explanation for them is that they are radio jet remnants from a formerly active galaxy that has turned off and left the remnant behind. Buoyancy in the relatively dense, X-ray emitting intracluster medium of a rich cluster of galaxies would foster the accumulation of radio source remnants near the edges of clusters. However, the typical lifetimes of the synchrotron emitting energetic electrons are so short $\left(\sim 10^{8}\right.$ years) that detection of these relics at radio frequencies requires a recent re-accelertion episode by shocks or compression [11] . So many more relics full of low energy electrons $\left(\gamma_{e} \simeq 10^{2}\right)$ may be present at the edges of clusters, or accumulate in the intercluster medium of superclusters, but still be invisible at radio frequencies.

To get an estimate of the length scale for a fossil reservoir, we assume that the transport of low energy cosmic ray electrons is regulated by feedback from self-excited waves, so that the streaming is effectively at the Alfven speed and along the direction of the magnetic field [12] [13]. The range from the point of origin for an energetic cosmic ray generated at time $t_{o}$ is then given by

$$
R=0.4\left(\frac{\mathrm{B}}{1 \mu \mathrm{G}}\right)\left(\frac{\mathrm{n}_{\mathrm{e}}}{3 \times 10^{-3} \mathrm{~cm}^{-3}}\right)^{-1 / 2}\left(\frac{t-t_{i}}{10^{10} \text { years }}\right) \mathrm{Mpc}
$$

So we expect a fossil reservoir to have a minimum scale size of $\sim \mathrm{Mpc}$. Another physical constraint on the fossil reservoir is an upper limit on the cosmic ray pressure. If this pressure from non-thermal particles in reservoir exceeds the thermal pressure of the surrounding gas, the reservoir will expand and generate prominent distortions in the intensity profiles of the X-ray emitting gas [14]. In the outer regions of X-ray emitting rich clusters where radio relics have been found, the thermal gas pressure is typically $\sim 10 \mathrm{eV} \mathrm{cm}^{-3}$. In the intercluster medium of a supercluster, pressure estimates using the thermal Sunyaev-Zeldovich effect [8] indicate a substantially lower thermal gas pressure of $\sim 10^{-2} \mathrm{eV} \mathrm{cm}^{-3}$.

\section{Prospects for Detecting Optical Emission from Fossil Reservoirs}

Given the simple physical constraints derived in the previous section, one can start to bound the expected optical surface brightness for a fossil cosmic ray electron reservoir using 3.5. Based 
on the streaming scale length of $\sim 1 \mathrm{Mpc}$ derived from equation 3.6, we assume that a 6 Billion year old fossil reservoir would span about $1 \mathrm{Mpc}$ along the line of sight. Then given the maximum sustainable cosmic ray pressure of $\sim 10 \mathrm{eV} \mathrm{cm}^{-3}$ in $\gamma_{e} \sim 50$ electrons in a reservoir at the edge of a rich clusters, we find a predicted bright limit for the surface brightness in the Sloan g-band of $S_{g} \sim 29.5 \mathrm{mag} \operatorname{arcsec}^{-2}$. Sources near this bright limit should be marginally detectable with the LANL array. However to reach such a high pressure would require an extreme interval of particle acceleration in the early Universe and preservation of those particles in a reservoir with low plasma density $n_{e}=10^{-5} \mathrm{~cm}^{-3}$. More realistic cosmic ray pressures of $\sim 0.1-1.0 \mathrm{eV} \mathrm{cm}^{-3}$ would yield $S_{g} \sim 32-34.5 \mathrm{mag} \operatorname{arcsec}^{-2}$.

\section{Discussion}

In section 3, we discussed the characteristic loss timescales for cosmic ray electrons in the local universe. But an important question for fossil reservoir development is: How old can the oldest particles be? To derive that age horizon, we calculate the redshift at which the characteristic lifetime for $\gamma_{e}=50$ electrons equals the look back time. The answer is somewhat dependent on the cosmological model. But if we use standard parameters for the $\Lambda \mathrm{CDM}$ cosmology $\left(H_{o}=\right.$ $\left.67.74 \mathrm{~km} \mathrm{~s}^{-1} \mathrm{Mpc}^{-1}, \Omega_{\mathrm{m}}=0.3089, \Omega_{\Lambda}=0.6817\right)[15]$ and the $(1+z)^{4}$ scaling of the CMB energy density, we find $\mathrm{z}=0.66$ and a maximum age of 6.3 Billion years for the optical inverse Compton emitting fossil electrons. The strong $\mathrm{z}$ dependence of the CMB radiation density also means that we expect brighter sources at higher $\mathrm{z}$ because the predicted optical surface brightness scales as $(1+z)^{4}$.

While the prospects for detecting fossil cosmic ray reservoirs with the LANL array are low, interest is growing in low surface brightness imaging and the technology is now moving forward rapidly. For example, techniques are now being developed for low surface brightness imaging with the Hubble Space Telescope that should reach $S \sim 32.5$ mag $\operatorname{arcsec}^{-2}$ [16]. We are therefore entering an era when it will be possible to search for fossil cosmic ray electron reservoirs that are impossible to find any other way.

\section{Acknowledgements}

The research presented in this paper was supported by the Laboratory Directed Research and Development program of Los Alamos National Laboratory.

\section{References}

[1] R. Abraham and P. van Dokkum, P. Ultra-Low Surface Brightness Imaging with the Dragonfly Telephoto Array PASP, vol. 126, pp. 55-69 (2014)

[2] R. Abraham et al., Future Prospects: Deep Imaging of Galaxy Outskirts using Telescopes Large and Small arXiv:1612.06415 (2016)

[3] N. Tanvir et al.,A gamma-ray burst at a redshift of $z=8.2$,Nature, Vol. 461, Issue 7268, pp. 1254-1257 (2009) 
[4] R.J. Gould, Energy loss of fast electrons and positrons in a plasma, Physica, vol. 60, pp. 145-154 (1972)

[5] V. Ginzburg and S. Syrovatskii, The Origin of Cosmic Rays, Pergamon Press, MacMillian 1964.

[6] M. Longair, High Energy Astrophysics, vol.2, Cambridge University Press, 1994.

[7] G. Blumenthal and R. Gould, Bremsstrahlung, Synchrotron Radiation, and Compton Scattering of High-Energy Electrons Traversing Dilute Gases, Rev. Mod. Phys., Vol. 42, 237 (1970)

[8] H. Tanimura et al., Detection of intercluster gas in superclusters using the thermal Sunyaev-Zeldovich effect, Astron. Astrophysics, Vol. 625, A67 (2019).

[9] T. Ensslin Diffuse thermal and relativistc plasma in galaxy clusters, arXiv:astro-ph/9906212 (1999)

[10] C.L. Carilli and G.B. Taylor, Cluster Magnetic Fields, Annu. Rev. Astron. Astrophys., vol. 40,pp.319-348 (2002)

[11] T. Ensslin et al., Cluster radio relics as a tracer of shock waves of the large scale structure formation Astronomy and Astrophysics, vol. 332, pp.395-409 (1998)

[12] E. G. Zweibel, The basis for comic ray feedback:Written on the wind Physics of Plasmas, vol. 24, 055402 (2017)

[13] T. Thomas and C. Pfrommer, Cosmic-ray hydrodynamics:Alfven-wave regulated transport of cosmic rays, MNRAS, vol. 485, Issue 3, p.2977-3008 (2019)

[14] W.T. Vestrand Coma-type radio halos and cluster X-ray Morphology,Astronomical Journal, vol. 87, 9 (1982)

[15] P. Ade et al. Planck 2015 results. XII. Cosmological parameters Astronomy and Astrophysics, vol. 594, id. A13, 63 pp. (2016)

[16] A. Borlaff et al. the missing light of the Hubble Ultra Deep Field Astronomy and Astrophysics, vol. 621, id. A133 (2019) 